\title{
Individual goal-directed intraoperative fluid management of initially hypovolemic patients for elective major urological surgery
}

\author{
Szturz P, Kula R, Tichy J, Maca J, Neiser J, Sevcik P \\ Department of Anesthesiology and Intensive Care Medicine, University Hospital Ostrava and Ostrava University, \\ Faculty of Medicine, Czech Republic. szturz@post.cz
}

\begin{abstract}
Background: The impact of different approaches to fluid management during intraoperative volume resuscitation in patients undergoing major elective surgery is poorly defined. We compared volume effectiveness of crystalloid and colloid substitution aimed to maintain the cardiac index (Cl) between 2.6 and $3.8 \mathrm{l} / \mathrm{min} /$ $\mathrm{m}^{2}$ as measured by transesophageal Doppler (TED).

Methods: A total of 115 urological patients were enrolled in the prospective randomized trial and then randomized into 2 groups, one with volume therapy based on crystalloids $(n=57)$ and the other with colloids $(n=58)$. A TED probe was inserted and then hemodynamic optimization (therapy with Ringer's solution or hydroxyethyl starch $6 \% 130 / 0.4$ and administration of vasoactive drugs) was started according to TED variables to maintain the $\mathrm{Cl}$ between 2.6 and $3.8 \mathrm{l} / \mathrm{min} / \mathrm{m}^{2}$.

Results: We observed high incidence of $\mathrm{Cl}<2.6 \mathrm{l} / \mathrm{min} / \mathrm{m}^{2}$ after induction of anesthesia $(75 \%)$ in both groups. There were no significant differences in demographic characteristics, ASA classification, length of surgery, estimated blood loss and the $\mathrm{Cl}$ during surgery. To maintain the $\mathrm{Cl}$ within the requested interval, significantly different amounts of crystalloids were needed as compared to colloid (median: $5000 \mathrm{ml}$ vs $1500 \mathrm{ml}$ ). In the CRY group, more patients were treated by vasodilatators (40.4 vs $20.7 \%$ ).

Conclusions: The study confirmed that crystalloids and colloids are effective in correcting flow-related perfusion abnormalities. The significant difference between volumes of crystalloids and colloids proved their different characteristics such as unequal distribution between compartments. The expansion of therapeutic algorithm by using vasoactive drugs allows us to avoid adverse events resulting from fluid overload (Tab. 1, Fig. 5, Ref. 35). Text in PDF www.elis.sk.

Key words: perioperative fluid management, crystalloids, colloids, transesophageal Doppler.
\end{abstract}

\begin{abstract}
Abbreviations: Acc - peak aortic blood flow acceleration, ASA score - American Society of Anesthesiologists score, CI - cardiac index, CRY group - crystalloid group, COL group - colloid group, CVP - central venous pressure, FFP - fresh frozen plasma HR heart rate, IV - intravenous application, LVETi - left ventricular ejection time index, MAP - mean arterial pressure, RBC - red blood cells, TED - transesophageal dopplerometry, TSVRi - total systemic vascular resistance index.
\end{abstract}

The main goals of fluid therapy are volume expansion and restoration and maintenance of adequate perfusion and oxygen delivery; this therapy should always be adjusted to individual hemodynamic goals. However, there are some clinical conditions in which volume therapy could be harmful, such as fluid overload, followed by tissue edema formation and increased

Department of Anesthesiology and Intensive Care Medicine, University Hospital Ostrava and Ostrava University, Faculty of Medicine, Czech Republic

Address for correspondence: P. Szturz, University Hospital Ostrava, 17. listopadu 1790, CZ-708 52 Ostrava, Czech Republic. Phone: +420.59.7372702, Fax: +420.59.7372762

Acknowledgements: We gratefully acknowledge Jiri Jarkovsky, PhD, from The Institute of Biostatistics and Analyses of Masaryk University, Brno, Czech Republic, for his contribution and data analysis. cardiac filling pressure. A common practice is co-administration of fluids and vasoactive agents, which is supposed to reduce the above risk.

Crystalloid and colloid solutions have, according to their pharmacokinetics, different distribution between body compartments. Older studies, which included patients with trauma and hemorrhagic shock, described the need to use approximately three times the amount of crystalloids compared with colloids for restoration of effective tissue perfusion during emergency fluid resuscitation $(1,2,3)$. These findings correspond with the traditional view of the fluid shift between the intravascular and interstitial spaces (4). However, some recently published studies comparing volume effectiveness of these solutions questioned some of the conclusions. According to the SAFE study, 1.16 to 1.3 times more saline solution than $4 \%$ albumin is needed to reach the same values of mean arterial pressure (MAP) in the first four days of hospitalization (5). Another study (major cardiac and vascular surgery) performed in the early postoperative period comparing volume effectiveness of crystalloids and different colloids observed that the adequate volume ratio would be $1.2: 1$ (6). Influence of the used vasoactive agents on global perfusion was not evaluated in these studies. Based on these results, some authors recommended reviewing the traditional opinions related to the ratio of crystalloid and colloid solutions used during volume resuscitation. 


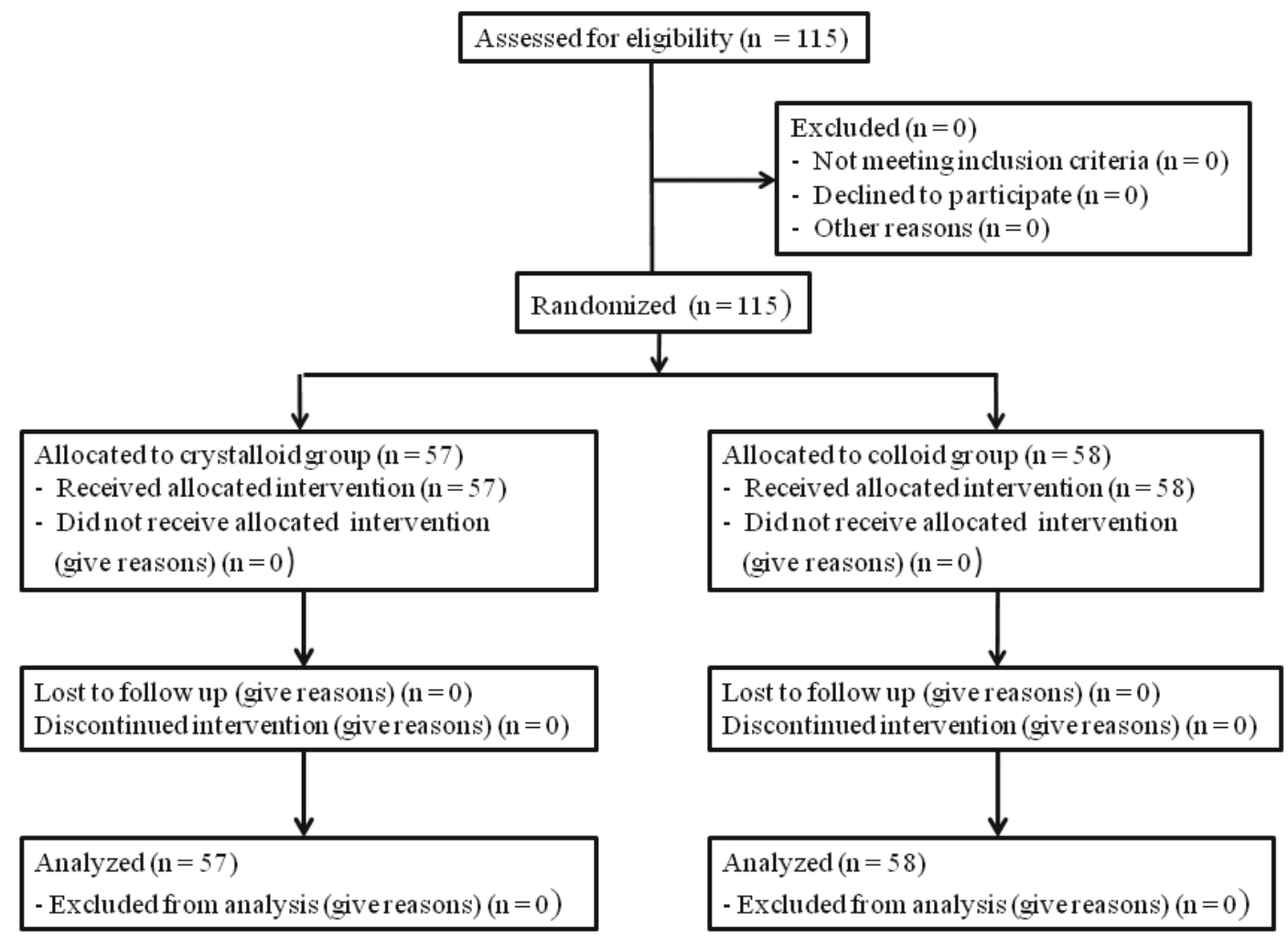

Fig. 1. Study CONSORT style chart.

The aim of this study was to compare volume effectiveness of colloids and crystalloids during major elective urological surgery using flow-related values derived by transesophageal Doppler (TED) monitoring. The therapeutic algorithm included the use of titrated doses of vasoactive agents in case there were some abnormalities in myocardial contractility and afterload values. Another goal was to find out if the type of solution used has influence on the frequency of postoperative complications and prognosis of the patient.

\section{Patients and methods}

A total of 115 consecutive patients undergoing elective major urological surgery have been enrolled in a prospective randomized clinical study (Fig. 1). The study was approved by the Institutional Research Ethics Committee and was carried out in operating theatres and an intensive care unit (ICU) of a tertiary hospital. Statement of the Ethics Committee confirms that all the activities and the structure of members of the Ethics Committee is in compliance with the international rules of conducting clinical trials. The exclusion criteria were age under 21 years, emergency surgery, pregnancy, severe cardiac or respiratory failure and expected duration of surgery less than 90 minutes. Informed consent was obtained from all patients enrolled in the study.

The patients were randomized and allocated according to the sequentially numbered, sealed opaque envelope technique. There were no restrictions or stratification in the randomization process. The research nurse assigning the patients to either the crystalloid (CRY, $n=57$ ) or colloid (COL, $n=58$ ) groups opened the allocation envelope immediately before induction of general anesthesia. After the induction, each patient obtained a TED probe (Hemosonic $^{\mathrm{TM}} 100^{\circledR}$, Arrow International, Inc., Reading, PA, USA). Then hemodynamic optimization (fluid therapy with Ringer's solution or hydroxyethyl starch $6 \%$ 130/0.4 and administration of vasoactive drugs) was started according to TED variables to maintain the cardiac index (CI) between 2.6 and $3.8 \mathrm{l} / \mathrm{min} / \mathrm{m}^{2}$. In each patient from any of the groups, the probe was inserted through the mouth to the distal third of the esophagus. According to the predefined therapeutic management algorithm (Fig. 2), fluids, inotropic support with dobutamine

(Dobutamin Lachema 250, Pliva-Lachema a.s., Brno, Czech Republic), and vasoactive support with noradrenaline (Noradrenalin Leciva, Zentiva, Czech Republic) or isosorbide dinitrate (Isoket roztok $0.1 \%$, Schwarz Pharma AG, Monheim, Germany) were used. To compare the volume efficacy of maintaining the CI in the normal range with crystalloids or colloids, Ringer's solution (Ringer's injection, Fresenius Kabi, Verona, Italy) for volume resuscitation was used in the CRY group and hydroxyethyl starch (HES, Voluven ${ }^{\circledR}$, Fresenius Kabi AG, Bad Homburg, Germany) in the COL group. Concentration of hemoglobin lower than $90 \mathrm{~g} / \mathrm{l}$ was an indication to start blood substitution. The supplementation of immeasurable fluid losses in the COL group was performed 


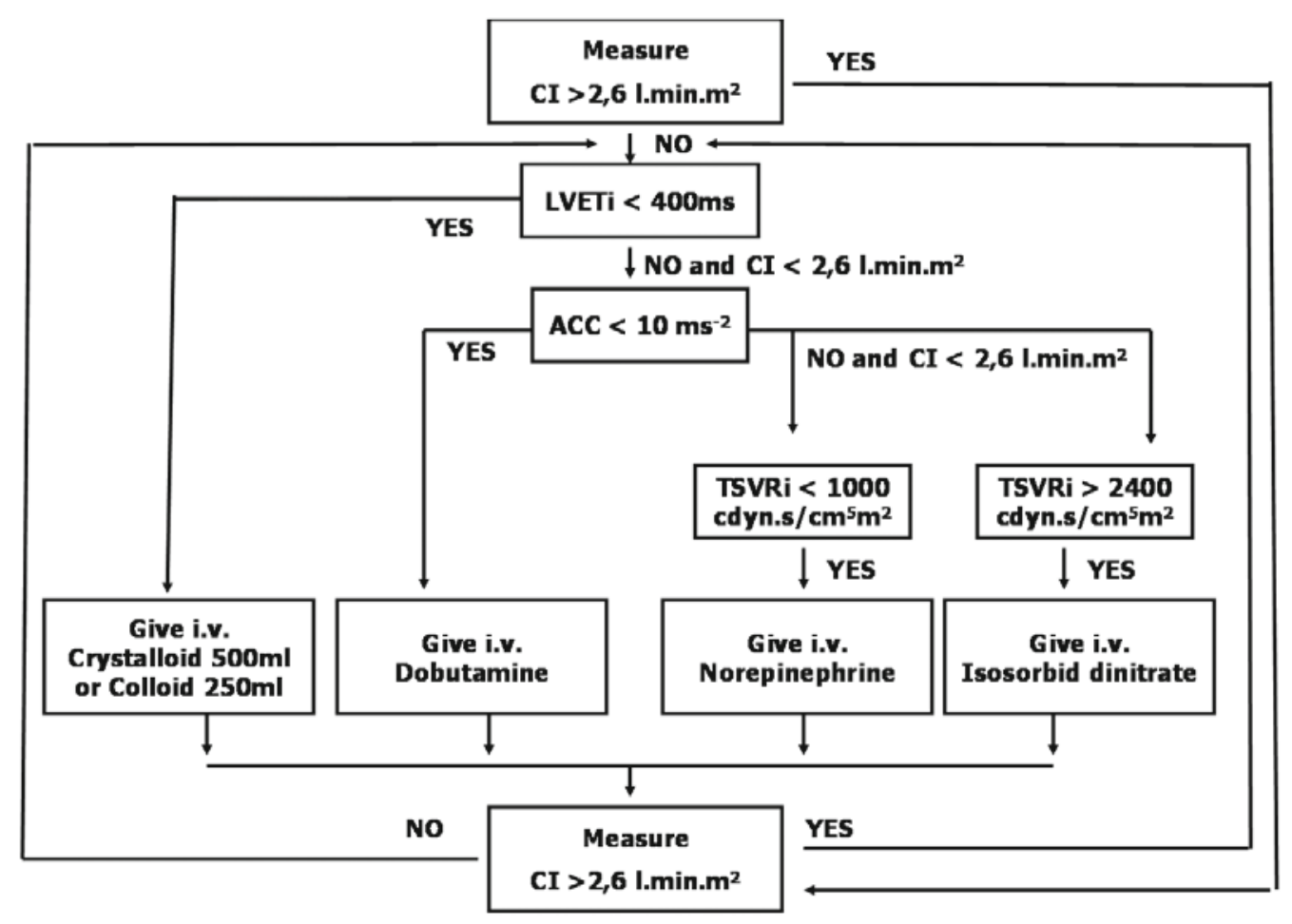

Fig. 2. Therapeutic algorithm.

with Ringer's solution $0.05 \mathrm{ml} / \mathrm{kg} / \mathrm{min}$. None of the patients was excluded after randomization.

All patients had their bowels prepared by enema and/or using phosphate solution in the evening before surgery. This therapy was extended by administration of bisacodyl (Fenolax ${ }^{\circledR}$, ICN Polfa, Rzeszow, Poland) and colonoscopy preparation diet in case of planned radical cystectomy. The patients were encouraged to drink water until midnight. Intravenous fluids, usually used overnight to minimize dehydration before surgery, were not administered due to local urological recommendations.

General anesthesia was induced with propofol and maintained with a balanced technique incorporating mixed nitrous oxide and oxygen, isoflurane with cisatracurium providing muscle relaxation. Sufentanil was used for analgesia at the anesthetist's discretion. The patients were intubated and ventilated to normocapnia throughout the operation. Standard monitoring included ECG, pulse oxymetry, capnography, and measurement of invasive arterial blood pressure. Prior to the operation, central venous catheter was introduced in 50 patients (88 \%) in the CRY group and 49 patients (84 \%) in the COL group. Intraoperative epidural analgesia was never used.

All patients enrolled in the study were admitted to a surgical ICU after the procedure and followed until discharge from the hospital. The following parameters were recorded: length of ICU stay, length of hospital stay, rate of postoperative complications and in-hospital mortality. The evaluation of postoperative complications was similar to the method used by Bennett-Guerrero and colleagues (7).
Primary endpoint was defined as the comparison of volume replacement efficiency of crystalloids and colloids during major urological surgery. This efficiency was measured by flow-related perfusion parameters derived by transesophageal Doppler (TED) monitoring. Secondary endpoints included postoperative complications, ICU length of stay, length of stay in hospital and mortality related to the type of used fluids.

For sample size estimation, $750 \mathrm{ml}$ difference in the amount of administered fluids (with a $5 \%$ significance level and $80 \%$ power, SD $1250 \mathrm{ml}$ ) was considered clinically important. A sample size of 45 patients in both groups (i.e. 90 patients) was necessary. For the particular sample size, variability and difference in the mean volume of intraoperatively administered fluids between the colloid and crystalloid groups, the power of the test was found to be almost $100 \%$. This power of the test is sufficient with respect to differences found by the study.

Data on patient characteristics, complications, and Doppler measurement were extracted from the (electronic) patient records. To ensure a proper execution of the trial and to monitor the progress, outcome, and patient safety during the trial, its progress and any occurring adverse events were discussed regularly with an expert team that was not involved in the randomization procedure or postoperative treatment of the patients.

Statistical analysis was carried out by an independent statistical institution. The Mann-Whitney U test was applied to compare continuous variables and the chi-square or Fisher's exact tests were used for categorical variables. The Wilcoxon matched pairs 

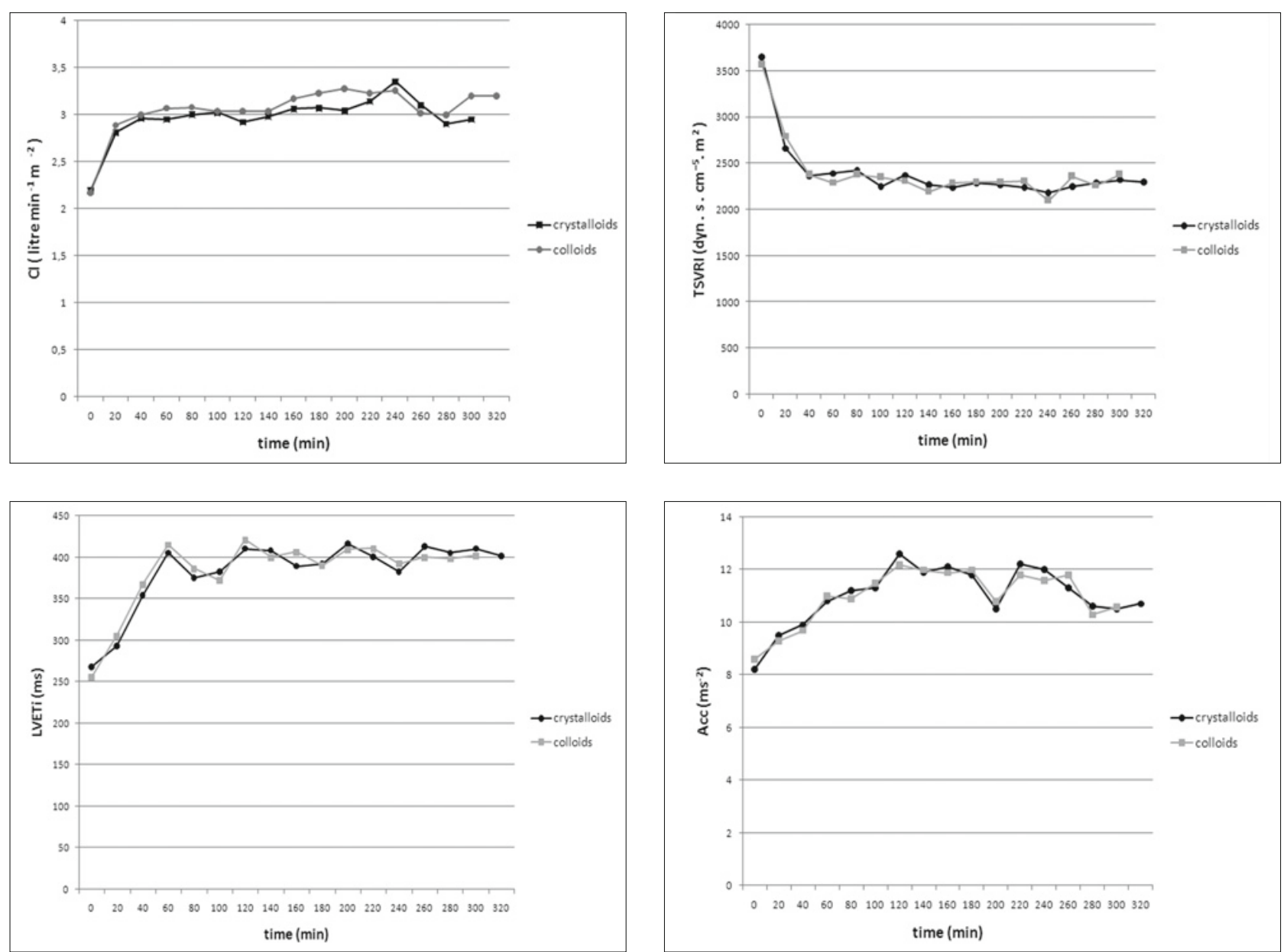

Fig. 3. Influence of crystalloids and colloids on flow-related hemodynamic variables during intraoperative period. Median of intraoperative values of CI, LVETi, Acc, TSVRi for all patients in the crystalloid and colloid group was without statistically significant differences.

Tab. 1. Patient characteristics and intraoperative course details.

\begin{tabular}{lcc}
\hline & Crystalloid group & Colloid group \\
\hline number of patients & 57 & 58 \\
\hline age in years & $61(27-87)$ & $63.5(22-93)$ \\
\hline male gender & $41(71.9 \%)$ & $42(72.4 \%)$ \\
\hline ASA score & $2(1-4)$ & $2(1-4)$ \\
\hline BMI (m2) & $2.0(1.5-2.6)$ & $2.0(1.7-2.7)$ \\
\hline surgery for tumor & $45(78.9 \%)$ & $46(79.1 \%)$ \\
\hline Type of surgery & & \\
$\quad$ nephrectomy & $20(35.1 \%)$ & $24(41.4 \%)$ \\
radical prostatectomy & $24(42.1 \%)$ & $23(39.7 \%)$ \\
$\quad$ cystectomy & $13(22.8 \%)$ & $11(19.0 \%)$ \\
\hline duration of surgery (min) & $155(110-385)$ & $150(115-370)$ \\
\hline estimated blood loss (mL) & $1100(100-4000)$ & $1000(100-3000)$ \\
\hline blood administrated (units/patient) & $0.9(0-6)$ & $0.3(0-3)^{*}$ \\
\hline FFP (units/patient) & $1.0(0-7)$ & $0.3(0-4)^{*}$ \\
\hline crystalloids administrated (mL) & $5000(2600-9000)$ & $700(200-1600)^{*}$ \\
\hline colloid administrated (mL) & 0 & $1500(400-3500)^{*}$ \\
\hline dobutamine & $75.4 \%$ & $67.2 \%$ \\
\hline norepinephrine & $5.3 \%$ & $0 \%$ \\
\hline isosorbide dinitrate & $40.4 \%$ & $20.7 \% *$ \\
\hline Values are presented as absolute (percentage) or median (interquartile range). * \\
$p<0.05$
\end{tabular}

test was adopted for the comparison of paired measurements of patients. A $p$ value of less than 0.05 was considered statistically significant.

\section{Results}

During the inclusion period 115 patients were potentially suitable for the study. After screening, all patients match all inclusion criteria. The flow chart of the number of patients at inclusion, during follow-up and available for analysis is shown in Figure 3. We therefore analyzed the results of all 115 patients who were included and had entered the protocol. Demographic data of all patients enrolled in the study are presented in Table 1. Statistical analysis did not show any differences in age (median: 61 years in the CRY group vs 63.5 years in the COL group), gender (males: $71.9 \%$ vs $72.4 \%$ ), body surface area (median: 2.0 vs 2.0 ), and estimated risk of anesthesia according to the ASA classification (median: $2.0 \mathrm{vs}$ 2.0). Most of the patients underwent surgery for a tumor (78.9\% in the CRY group vs $79.3 \%$ in the COL group). The most frequent surgery was radical prostatectomy ( $42.1 \%$ vs $39.7 \%$ ), followed by nephrectomy (35.1\% vs $41.4 \%$ ) and cystectomy (22.8\% vs $19 \%$ ). 


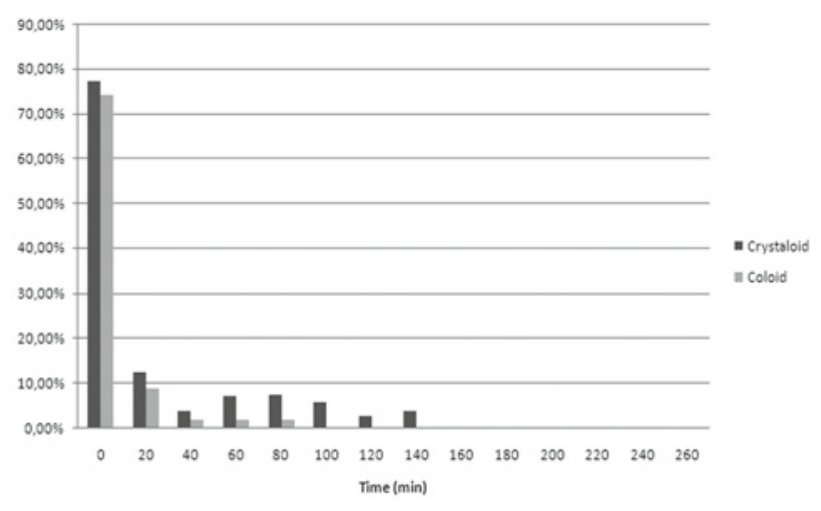

Fig. 4. Proportion of patients with cardiac index $<2.61 / \mathrm{min} / \mathrm{m}^{2}$ during surgery in both groups of patients.

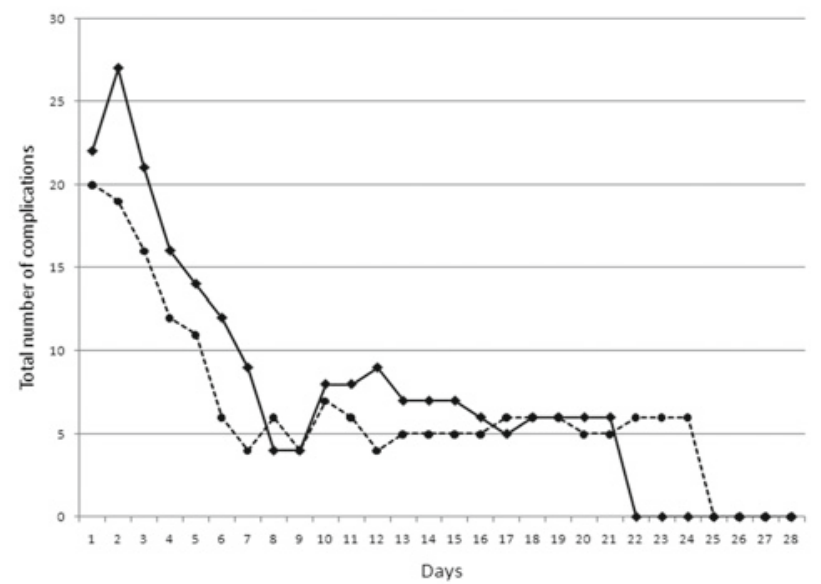

Fig. 5. Total number of postoperative complications in both groups of patients. Number of complications (hemodynamic, respiratory, renal, GIT, coagulation and neurology) did not reach statistical significance during ICU hospitalization.

Data concerning surgery are presented also in Table 1. All surgeries lasted at least 90 minutes (median: 155 minutes in the CRY group vs 150 minutes in the COL group). To maintain the CI within the requested interval, significantly different amounts of crystalloids compared to colloids were needed (median: 5000 $\mathrm{ml}$ vs $1500 \mathrm{ml}, \mathrm{p}=0.001$, respectively).Total amount of fluids (except for red blood cells and fresh frozen plasma) was $5000 \mathrm{ml}$ in the CRY group vs $2200 \mathrm{ml}$ in the COL group. The number of intraoperatively administered blood units was also higher in CRY group than in the COL group (red blood cells: median 0.9 vs $0.3 \mathrm{p}$ $=0.018$; fresh frozen plasma: median 1.0 vs $0.3, \mathrm{p}=0.006$, respectively). However, the differences in administered blood units and fresh frozen plasma within the day of surgery were not significant. Significant differences were found in the use of vasoactive agents during the surgery. In the CRY group, more vasodilators (40.4\% vs $20.7 \%$ ) were used. The differences in amounts of administered inotropic and vasoactive drugs were not statistically significant. There were no significant differences in the values of left ventricular ejection time index (LVETi), peak aortic blood flow acceleration (Acc) and total systemic vascular resistance index (TSVRi) between both study groups. Dynamic changes in LVETi, Acc and TSVRi are shown in Figure 4. The time course of measured values of the CI describing the actual achievement of the target in both groups is shown in Figure 5. More than $70 \%$ of patients in both groups had the initial CI lower than $2.6 \mathrm{l} / \mathrm{min} / \mathrm{m}^{2}$ (median: 2.17 vs 2.19). However, the rate dropped to $10 \%$ of patients after 20 minutes and always stayed lower than $5 \%$ of patients during the further course of the procedure.

The dynamics of intraoperative catecholamine dosage also suggested that the initial higher doses of dobutamine and isosorbide dinitrate could be, after achieving the desired cardiac index, lowered and subsequently even discontinued. Despite the statistically significant differences in the frequency of isosorbide dinitrate administration between the two groups, continuous administration of the infusion was terminated (after achievement of hemodynamic values according to protocol) in all patients before their surgery was completed. Besides, there were no significant differences in the values of mean arterial pressure, central venous pressure and heart rate between both study groups, with all of them being within the normal interval (Fig. 5).

With regard to postoperative complications, there was a statistical significance related only to gastrointestinal tract dysfunction in the CRY group (31.6 \%) vs the COL group (15.5 \%; $\mathrm{p}=0.05)$. The number of complications in hemodynamics, renal, respiratory and hematological parameters was not statistically significant. The overall amount of complications during ICU stay was not significantly different. Also the length of ICU stay, the length of hospital stay and mortality were not significantly different.

\section{Discussion}

The primary goal of fluid therapy is reduction of plasma volume deficit and thus to achieve an adequate CI, tissue perfusion and oxygen delivery. However, fluid overload can cause excessive extravascular fluid retention, pulmonary congestion and other complications $(8,9)$. Vasoactive drugs may effectively prevent the complications. This rules have been derived from complex approach to Frank-Starling law (not only preload changes, but also afterload and contractility are responsible for curve shift) (10). Therefore, optimal management might be a combination of fluid and vasoactive therapy which maintains the CI index within the normal interval without excessive elevation of cardiac filling pressure or preventing the development of interstitial edema

The causes of perioperative hypovolemia are multifactorial, including preoperative fasting and bowel preparation $(11,12)$. Hypovolemia induces peripheral vasoconstriction and microcirculatory compromise due to bar receptor reflex-induced increase in sympathetic activity and release of catecholamine, and is an important factor contributing to organ failure.

After introduction to general anesthesia, worsening of global hemodynamic parameters is observed, especially preload, which causes reduction of the CI and parallel elevation of systemic vascular resistance (13). However, traditional parameters such as heart rate (HR), mean arterial pressure (MAP) and central venous pressure (CVP) are often normal (14). Use of anesthetics with 


\section{$653-659$}

cardiodepressive and vasodilator effects (15) or acute blood loss further enhance the negative impact on tissue perfusion.

There are no randomized controlled trials which would sufficiently evaluate interaction between the amounts of administered fluids and vasoactive agents used to reduce hypoperfusion abnormalities in relation to outcome of patients undergoing elective major surgery, trauma, sepsis or burns (16). Recommendation for volume resuscitation of early hypoperfusion abnormalities is based on identification of real intravascular volume deficiency and also on knowledge about different biological and physicochemical properties of crystalloid and colloid solutions such as the length of volume-expansion effect, hemorrheologic properties (with influence on vascular integrity and immunologic response) and simultaneously reduction of potential adverse effects of any administered fluids $(8,17)$.

Perioperative fluids amount should be directed by reaching individual hemodynamic goals. Administration of crystalloids is sufficient for reaching euvolemia in almost all clinical situations. Administration of colloids (both natural and synthetic) should always be justified by real need of fast volume replacement and measurement of dynamic flow parameters.

From healthy volunteer studies and also from clinical practice, it is clear that for achievement of the same equivalent plasma volume expansion effect, 3-4 times the amount of crystalloids is needed as compared to colloids $(18,19)$. In the SAFE study, during the first 4 days of hospital stay at an intensive care unit, the crystalloid to $4 \%$ albumin ratio was found to range from 1.16 to 1.4 : 1. Similar results (crystalloid: colloid; 1:1.45) were noted in septic patients in the VISEP study where the goals of hemodynamic optimization were adjustments in HR, MAP, CVP and mixed venous oxygen saturation $(5,20)$. Nevertheless, a recent study performed in an early postoperative period showed that while using the same amounts of different solutions, colloids are more effective in increasing cardiac filling, output and oxygen delivery than administration of Ringer's solution (21). Interaction between amounts of administered fluid and vasoactive agents was not evaluated.

The results of our study confirmed the assumption that hypovolemia can be frequently found in patients undergoing major elective surgery $(13,22)$. We also verified high significance of flow-related parameters for detection of hypoperfusion abnormalities $(23,24,25)$ while using TED. The study proved that both crystalloids and colloids are efficient tools correcting early flow hemodynamic abnormalities, but there was no statistically significant difference in some important outcome parameters such as the overall frequency of postoperative complications, length of ICU stay, overall length of hospitalization and in-hospital mortality. On the other hand, statistically significant differences in the amount of administered crystalloid and colloid solutions suggest different expansion characteristics. The extension of the therapeutic algorithm by the use of vasoactive drugs aids in avoiding potential adverse events resulting from fluid overload $(26,27)$. Effectiveness of therapeutic algorithm was proven in 230 surgical patients. Mortality, rate of perioperative complications, length of hospital stay were all lower in group with optimization of flow parameters compared to the group with standard perioperative monitoring only (28). We also verified their influence on correction of altered parameters of global perfusion, which finally helped us adjust the infused amounts of crystalloid and colloid solutions more effectively. Higher frequency of gastrointestinal tract complications in the group of patients using crystalloids reminds us of possible adverse effects resulting from administration of these solutions (29). Gut edema may be associated with postoperative gastrointestinal dysfunction, impairment of tissue oxygenation and increased intraabdominal pressure (7, 30-33). These results could be affected by different strategy of fluid management in ICU, where no postoperative protocol was standardized.

We are aware of the limits of the study, namely the small number of patients and specificity of major urologic surgery, which do not allow to exactly apply the results to another subpopulation of critically ill patients. Moreover, the used flow parameters to guide hemodynamic optimization could not sufficiently define preload, contractility and afterload. Nonetheless, we assume that these flow parameters are more accurate in titration of the amount of administered fluid and vasoactive drugs in the intraoperative period and have better correlation to outcome, in comparison with the traditional parameters (HR, MAP and CVP) $(34,35)$.

\section{Conclusion}

Despite considerable attention dedicated to perioperative medicine and significant advances in technology of perioperative hemodynamic monitoring devices, there are no standardized procedures and protocols recommending the dosage of fluids and vasoactive drugs. Early detection of intravascular volume deficiency by flow-related parameters is an effective tool in amelioration of hypoperfusion abnormalities. In these situations, both crystalloid and colloid solutions may still be administered together with vasoactive drugs to avoid adverse effects generally related to fluid therapy. To prove this in other groups of patients, more extensive randomized controlled trials are needed. Definition of hemodynamic parameters and creating algorithms allowing early and adequate therapeutic intervention therefore remain a great challenge for future perioperative research.

\section{References}

1. Shoemaker WC, Schluchter M, Hopkins JA, Appel PL, Schwartz S, Chang PC. Comparison of the relative effectiveness of colloids and crystalloids in emergency resuscitation. AM J Surg 1981; 42: 73-74.

2. Modig J. Advantages of dextran 70 over Ringer acetate solution in shock treatment and in prevention of adult respiratory distress syndrome. A randomized study in man after traumatic-haemorrhagic shock. Resuscitation 1983; 10: 219-226.

3. Perel P, Roberts I. Colloids versus crystaloids for fluid resuscitation in critically ill patients. Cochrane Database Syst Rev 2007; (online): CD 000567.

4. Starling EH. On the absorption of fluids from the convective tissue spaces. J Physiol (London) 1896; 19: 312.

5. Finfer S, Bellomo R et al. A comparison of albumin and saline for fluid resuscitation in the intensive care unit. N Engl J Med 2004; 350: 2247-2256. 
6. Verheij J, van Lingen A, Beishuzien A et al. Cardiac response is greater for colloid then saline fluid loading after cardiac or vascular surgery. Intensive Care Med 2006; 32: 1030-1038.

7. Bennett-Guerrero E, Welsby I, Dunn TJ, Young LR, Wahl TA, Diers TL, Phillips-Bute BG, Newman MF, Mythen MG. The use of a postoperative morbidity survey to evaluate patients with prolonged hospitalization after routine, moderate-risk, elective surgery. Anesth Analg 1999; 89: 514-519.

8. Roberts I, Alderson $\mathbf{P}$ et al. Colloid versus crystalloids for fluid resuscitation in critically ill patients. Cochrane Database Syst Rev 2004; 4: CD000567.

9. Holte K, Sharrock NE et al. Pathophysiology and clinical implications of perioperative fluid excess. Br J Anaesth 2002; 89: 622-632.

10. Starling EH. The LINACRE lecture on the law of the heart. (Cambridge,1915) London, 1918.

11. Holte K, Kehlet T. Compensatory fluid administration for preoperative dehydration - does it improve outcome? Acta Anaesthesiol Scand 2002; 46: 1089-1093.

12. Pernicky M, Murin J, Labas P. Perioperative cardiovascular and noncardiovascular risk in patiens with colorectal cancer. Bratisl Lek Listy 2007; 108 (6): 246-250.

13. Junghans $\mathbf{T}$, Neuss $\mathbf{H}$ et al. Hypovolemia after traditional preoperative care in patients undergoing colonic surgery is underrepresented in conventional hemodynamic monitoring. Int J Colorectal Dis 2006; 21(7): 693-697.

14. Lobo SM, Lobo FR, Polachini CA, Patini DS, Yamamoto AE, de Oliveira NE, Serrano P, Sanches HS, Spegiorin MA, Queiroz MM, Christiano AC Jr, Savieiro EF, Alvarez PA, Teixeira SP, Cunrath GS. Prospective, randomized trial comparing fluids and dobutamine optimization of oxygen delivery in high-risk surgical patients. Crit Care 2006; 10: R72.

15. Cohn LH, Edmunds LH Jr (Eds). Cardiac Surgery in the Adult. New York: McGraw-Hill, 2003: 249-281.

16. Bellamy MC. Wet, dry or something else? Br J Anesth 2006; 97: 755-757.

17. Hartog Ch, Reinhart K. Contra: Hydroxyethylstarch solutions are unsafe in critically ill patients. Intensive Care Med 2009; 35: 1337-1342.

18. Berg S, Golster M, Lisander B. Albumin extravasation and tissue washout of hyaluronan after plasma volume expansion with crystalloid or hypooncotic colloid solutions. Acta Anaesthesiol Scand 2002; 46: 166-172.

19. Lobo DN, Stanga Z, Aloisyus MM et al. Effect of volume loading with 1 liter intravenous infusions of $0.9 \%$ saline, $4 \%$ succinylated gelatine (Gelofusine) and 6\% hydroxyethyl starch (Voluven) on blood volume and endocrine responses: a randomized, three-way crossover study in healthy volunteers. Crit Care Med 2010; 38: 464-470.

20. Brunkhorst FM, Engel $\mathbf{C}$ et al. Intensive insulin therapy and pentastarch resuscitation in severe sepsis. N Engl J Med 2008; 354: 125-139.

21. Gondos T, Marjanek Z, Ulakcsai Z et al. Short term effectiveness of different volume replacement therapies in postoperative hypovolaemic patients. Eur J Anaesthesiol 2010; 2: 794-800.
22. Bungaard-Nielsen M, Ruhnau B et al. Flow-related techniques for preoperative goal directed fluid optimization. Br J Anesth 2007; 98: 38-44.

23. Chytra I, Pradl R, Bosman R, Pelnar P, Kasal E, Zidkova A. Esophageal Doppler-guided fluid management decreases blood lactate levels in multiple-trauma patients: a randomized controlled trial. Crit Care 2007; 11: R24

24. Hillova Mannova J, Silhart Z, Sevcik P, Prokes A. Perioperative haemodynamic monitoring by oesophageal Doppler improves outcome of patients with abdominal aortic aneurysm repair. Bratisl Lek Listy 2013; 114 (2): 78-83.

25. Conway DH, Mayall R, Abdul-Latif MS, Gilligan S, Tackaberry C. Randomised controlled trial investigating the influence of intravenous fluid titration using oesophageal Doppler monitoring during bowel surgery. Anaesthesia 2002; 57: 845-849.

26. Vane LA, Prough DS, Kinsky MA et al. Effects of different catecholamines on the dynamics of volume expansion of crystalloid infusion. Anesthesiology 2004; 101: 1136-1144.

27. Bonazzi M, Gentile F, Biasi GM et al. Impact of perioperative haemodynamic monitoring on cardiac morbidity after major vascular surgery in low risk patients. A randomised pilot trial. Eur J Vasc Surg 2002; 23: 445-451.

28. Szturz P, Kula R, Maca J, Tichy J, Jahoda J. Intraoperative effectiveness of crystalloid and colloid volume substitution in patients undergoing elective major urological surgery by maintenance of the cardiac index within normal range. Crit Care 2011; 15 (Suppl 1): P 85.

29. Lobo SM, Orrico SPR, Queiroz MM, Contrim LM, Cury PM. Comparison of the effects of lactated Ringer solution with and without hydroxyethyl starch fluid resuscitation on gut edema during severe splanchnic ischemia Braz J Med Biol Res 2008; 41 (7): 634-639.

30. Balogh Z, McKinley BA, Cocanour CS, Kozar RA, Valdivia A, Sailors RM et al. Supranormal trauma resuscitation causes more cases of abdominal compartment syndrome. Arch Surg 2003; 138: 637-642.

31. Mythen MG. Postoperative gastrointestinal tract dysfunction. Anesth Analg 2005; 100: 196-204.

32. Baum TD, Wang H, Rothschild HR, Gang DL, Fink MP. Mesenteric oxygen metabolism, ileal mucosal hydrogen ion concentration, and tissue edema after crystalloid or colloid resuscitation in porcine endotoxic shock: comparison of Ringer's lactate and $6 \%$ hetastarch. Circulat Shock 1990; 30: 385-397.

33. Junghans T, Boohm B et al. Conventional monitoring and intravascular measurement can lead to different therapy after upper gastrointestinal tract surgery. Intens Care Med 2002; 28: 1273-1275.

34. Buhre W, Weyland A et al. Changes in central venous pressure and pulmonary capillary wedge pressure do not indicate changes in right and left heart volume in patients undergoing coronary artery bypass surgery. Eur J Anaesthesiol 1999; 16: 11-17.

35. Shoemaker WC, Wo CC, Thangathurai D, Velmahos G, Belzberg H, Asensio JA, Demetriades D. Hemodynamic patterns of survivors and nonsurvivors during high risk elective surgical operations. World J Surg 1999; 23: 1264-2670. 